\title{
A nomogram-based immunoprofile predicts clinical outcomes for stage II and III human colorectal cancer
}

\author{
LINGXIONG WANG $^{1}$, NIJIA CHANG ${ }^{2}$, LIANGLIANG WU ${ }^{1}$, JINFENG LI $^{1}$, LIJUN ZHANG ${ }^{1}$, \\ YIN CHEN $^{1}$, ZHOU ZHOU $^{1}$, JIANQING HAO ${ }^{3 *}$, QIONG WANG ${ }^{4 *}$ and SHUNCHANG JIAO ${ }^{5 *}$ \\ ${ }^{1}$ Institute of Oncology, The Fifth Medical Centre, Chinese PLA General Hospital; ${ }^{2}$ Department of Oncology, \\ The Second Medical Centre, Chinese PLA General Hospital, Beijing 100853; ${ }^{3}$ Department of Pneumology, \\ Qingyang People's Hospital, Qingyang, Gansu 745000; ${ }^{4}$ Department of Pathology, The First Medical Centre, \\ Chinese PLA General Hospital; ${ }^{5}$ Department of Oncology, The Fifth Medical Centre, \\ Chinese PLA General Hospital, Beijing 100853, P.R. China
}

Received April 15, 2021; Accepted September 17, 2021

DOI: $10.3892 / \mathrm{mco} .2021 .2419$

\begin{abstract}
An immunoscore for colorectal cancer (CRC) has higher prognostic significance than the TNM staging system. However, the tumor immune microenvironment contains various components that affect clinical prognosis. Therefore, a broader range of immune markers is required to establish an accurate immunoprofile to assess the prognosis of patients with CRC. Using immunohistochemistry combined with multispectral immunohistochemistry and objective assessments, the infiltration of four immune cell types $\left(\mathrm{CD}^{+} / \mathrm{CD}^{+} /\right.$forkhead box $\mathrm{p}^{+} / \mathrm{CD}^{3} 3^{+}$cells), as well as the expression of six co-signaling molecules [programmed cell death 1 (PD1) ligand 1/PD1/T-cell immunoglobulin mucin family member $3 /$ lymphocyte-activating $3 /$ tumor necrosis factor receptor superfamily, member $4 /$ inducible T-cell costimulator] and indoleamine 2,3-dioxygenase 1 were investigated in two independent cohorts of CRC. The patients' overall survival (OS) was evaluated using the Kaplan-Meier method. Using the Cox proportional hazards model, independent prognostic factors of patients were assessed and a nomogram-based
\end{abstract}

Correspondence to: Professor Shunchang Jiao, Department of Oncology, The Fifth Medical Centre, Chinese PLA General Hospital, 28 Fuxing Road, Beijing 100853, P.R. China

E-mail: jiaosc@vip.sina.com

*Contributed equally

Abbreviations: OS, overall survival; C-index, concordance index; ROC, receiver operating characteristic; CRC, colorectal cancer; AJCC, American Joint Committee on Cancer; UICC-TNM, International Union Against Cancer-TNM; Tregs, regulatory T cells; MDSCs, myeloid-derived suppressor cells; IDO1, indoleamine 2,3-dioxygenase 1; CK, cytokeratin; IHC, immunohistochemistry; TCs, tumor cells; ICs, immune cells; mIHC, multiplex IHC; TME, tumor microenvironment

Key words: colorectal cancer, nomogram, immunoprofile, $\mathrm{CD} 8^{+} \mathrm{TIL}, \mathrm{CD} 33^{+} \mathrm{MDSC}$ immunoprofile system was developed. The predictive ability of the nomogram was determined using a concordance index (C-index) and calibration curve. To facilitate clinical application, a simplified nomogram-based immunoprofile was constructed. Using receiver operating characteristic (ROC) analysis, the predictive accuracy for OS was compared between the immunoprofile and the TNM staging system for patients with stage II/III CRC. According to multivariate analysis for the primary cohort, independent prognostic factors for $\mathrm{OS}$ were $\mathrm{CD}^{+}$tumor-infiltrating lymphocytes, CD33+ myeloid-derived suppressor cells and TNM stage, which were included in the nomogram. The $\mathrm{C}$-index of the nomogram for predicting OS was 0.861 (95\% CI: 0.796-0.925) for the internal validation and 0.759 (95\% CI: 0.714-0.804) for the external validation cohort. The simplified nomogram-based immunoprofile system was able to separate same-stage patients into different risk subgroups, particularly for TNM stage II $(\mathrm{P}<0.0001)$ and III $(\mathrm{P}=0.0002)$ patients. Pairwise comparison of ROC curves for the immunoprofile and TNM stage systems for patients with stage II/III CRC revealed statistically significant differences $(\mathrm{P}=0.046)$ and the $\mathrm{Z}$-statistic value was 1.995 . In conclusion, the nomogram-based immunoprofile system provides prognostic accuracy regarding clinical outcomes and is a useful supplement to the TNM staging system for patients with stage II/III CRC.

\section{Introduction}

The prognosis of patients with colorectal cancer (CRC) after surgical resection is based on the American Joint Committee on Cancer (AJCC)/International Union Against Cancer (UICC)-TNM staging system $(1,2)$. However, this anatomy-based system gives useful but insufficient prognostic information, particularly for prognosis assessments in patients with UICC stage II and III CRC (2-5).

In numerous solid tumor types, such as $\mathrm{CRC}$, accumulating studies have indicated that the invasion of immune factors, depending on the type, density and location of infiltration (6) in the tumor microenvironment, has a profound impact on the prognosis of cancer patients (7-10). Specifically, a high density 
of adaptive immune cells infiltrating the core and invasive margin of the tumor was associated with a favorable prognostic effect for disease-free survival and overall survival (OS) in patients with early and advanced-stage CRC $(11,12)$. Thus, an immunoscore based on the density and location of $\mathrm{CD} 3 / \mathrm{CD}^{+}$ lymphocytes was used as a supplementary component for CRC classification (13).

However, the tumor immune microenvironment is complex and involves multiple immune cell types (14), as well as co-signaling molecules and immunomodulatory factors $(15,16)$, which may produce both pro- and antitumor functions. Multiple immune effector cells, such as CD8+TIL or $\mathrm{CD} 4^{+} \mathrm{TIL}$, and immunosuppressive cells, such as regulatory $\mathrm{T}$ (Treg) cells or myeloid-derived suppressor cells (MDSCs), are reportedly present at the tumor site (14). Immune cell subtypes have demonstrated differential prognostic value depending on the histological type of cancer (17). In melanoma, as well as colorectal and breast cancer, high CD8 ${ }^{+}$TIL infiltration was strongly correlated with favorable clinical outcome (8). However, the role of Treg cells in CRC is controversial, as Foxp ${ }^{+}$Treg infiltration indicated favorable prognosis in certain studies (18-21). Furthermore, MDSCs are a heterogeneous collection of immature myeloid cells that exhibit pathological activation and display potent immunosuppressive activity in the tumor microenvironment (22). Recently, the prognostic value of MDSCs was studied in different types of cancer (23-25), but the prognostic significance of tumor-infiltrating MDSCs in CRC has remained to be fully determined.

To promote or suppress T-cell activation, co-signaling molecules may be classified as co-stimulators or co-inhibitors (15). Programmed cell death 1 (PD1)/PD1 ligand 1 (PD-L1), the most extensively studied co-inhibitors, initiated a new wave of cancer immunotherapy (26). Within the past several years, PD1 or PD-L1 inhibitors have been successively approved as first- or second-line therapies for various solid and hematological tumors, including metastatic CRC with mismatch-repair-deficiency or microsatellite instability-high $(27,28)$. Furthermore, co-inhibitors such as lymphocyte-activating 3 (LAG3) and T-cell immunoglobulin mucin family member 3 (TIM3), as well as co-stimulators tumor necrosis factor receptor superfamily, member 4 (OX40) and inducible T-cell costimulator (ICOS), either alone or in combination with the PD1/PD-L1 pathway, have been tested in clinical trials for various advanced malignancies, including CRC $(16,29)$. However, the prognostic value of these co-signaling molecules remains controversial and has remained to be fully elucidated. Another factor of interest is indoleamine 2,3-dioxygenase 1 (IDO1), an immunomodulatory factor expressed in immune cells and various tumor cells (TCs) that potently mediates immunosuppressive effects in cancer (30). Whether IDO1 expression in different cells offers prognostic value for CRC warrants investigation.

Recently, a visualized nomogram model was developed for a variety of cancer types, which involved more parameters and had better survival prediction ability than TNM staging $(24,31,32)$. Thus, a wide range of immune markers may be used to establish an accurate immunoprofile to evaluate the prognosis of patients with CRC. In the present study, the infiltration of four immune cell types (CD8 ${ }^{+} \mathrm{TIL}, \mathrm{CD} 4^{+} \mathrm{TIL}$, Foxp3 ${ }^{+}$Treg and $\left.\mathrm{CD}_{3} 3^{+} \mathrm{MDSC}\right)$, as well as the expression of four co-inhibitors (PD1/PD-L1/TIM3/LAG3), two co-stimulators
(OX40/ICOS) and the immunomodulatory factor IDO1 were evaluated in CRC. Subsequently, the independent prognostic impact of the above variables was ascertained and a nomogram-based immunoprofile for CRC was established. The nomogram-based immunoprofile system provides diagnostic accuracy in the prognosis of clinical outcomes and is a useful supplement to TNM staging for patients with stage II/III CRC. It is expected that the present results will improve the ability of clinicians to distinguish different clinical outcomes in patients with CRC and the same TNM stage, particularly for TNM stage II/III patients.

\section{Materials and methods}

Study population and selection criteria. A retrospective study was performed on a primary cohort of 96 patients who underwent surgical resection of primary CRC at the First Medical Centre of Chinese PLA General Hospital (Beijing, China) between April 2010 and November 2013, with a mean follow-up time of $63.1 \pm 23.7$ months. The inclusion criteria were as follows: No history of neoadjuvant treatment, complete resection of colorectal tumors, histologic diagnosis of colorectal carcinoma and administration of standardized combination chemotherapy if relapsed. The exclusion criteria were as follows: Other malignancies, missing clinicopathological and follow-up information, and perioperative mortality. Using the same criteria, from January 2009 to May 2010, an independent validation cohort of consecutive patients with CRC $(\mathrm{n}=153)$, who were followed up for a mean of 50.4 \pm 26.8 months, was evaluated at Qingyang People's Hospital (Qingyang, China). All patients were classified using the AJCC 8th TNM staging system. These studies complied with ethical approval processes and were approved by the institutional review board the Chinese PLA General Hospital (Beijing, China). The clinical characteristics of the two cohorts are summarized in Table I.

Immunohistochemistry (IHC) and pathologic assessment. IHC staining of immune cell markers (CD4, CD8, Foxp3 and CD33) and other immunological markers (PD-L1, PD1, LAG3, TIM3, OX40, ICOS and IDO1) was performed as described previously (24), with slight modifications. In brief, after antigen retrieval and blocking of endogenous peroxidase, as well as blocking of non-specific binding sites, $3-\mu \mathrm{m}$-thick tissue sections were incubated with specific primary antibodies at $4^{\circ} \mathrm{C}$ overnight. Subsequently, the sections were incubated for 30 min with HRP-labeled rabbit/mouse secondary antibody (Gene Tech) at $37^{\circ} \mathrm{C}$. The primary antibodies used for IHC are listed in Table SI.

Immunostaining results were examined by two pathologists independently blinded to any information on the clinicopathological features of the patients using a semi-quantitative score. The relative percentage of positive cells for each marker was calculated using the mean value of five randomly selected high-magnification (x200) fields on full slides. The analysis of CD33 and the immunological markers, such as LAG3, TIM3, OX40 and ICOS, was performed on the entire tumor region (e.g., parenchyma and mesenchyme). The other markers (CD4/CD8/Foxp3/PD-L1/PD1/IDO1) were assessed in both the tumor parenchyma and mesenchyme regions. The assessment 
Table I. Basic clinical and pathological features of the patients from the two cohorts.

\begin{tabular}{|c|c|c|c|}
\hline Parameter & Primary cohort $(n=96)$ & Validation cohort $(n=153)$ & P-value \\
\hline Age, years & & & 0.191 \\
\hline Median & 62.5 & 66 & \\
\hline Range & $24-90$ & $22-95$ & \\
\hline Sex & & & 0.240 \\
\hline Male & $63(65.6)$ & $89(58.2)$ & \\
\hline Female & $33(34.4)$ & $64(41.8)$ & \\
\hline Tumor location & & & 0.108 \\
\hline Colon & $62(64.6)$ & $83(54.2)$ & \\
\hline Rectum & $34(35.4)$ & $70(45.8)$ & \\
\hline Histological type & & & 0.270 \\
\hline Adenocarcinoma & $94(97.9)$ & $144(94.1)$ & \\
\hline Other $^{\mathrm{a}}$ & $2(2.1)$ & $9(5.9)$ & \\
\hline Vascular invasion & & & 0.505 \\
\hline Yes & $7(7.3)$ & $8(5.2)$ & \\
\hline No & $89(92.7)$ & $145(94.8)$ & \\
\hline Grade & & & 0.705 \\
\hline G1 & $5(5.2)$ & $5(3.3)$ & \\
\hline $\mathrm{G} 2$ & $74(77.1)$ & $123(80.4)$ & \\
\hline G3 & $17(17.7)$ & 25 (16.3) & \\
\hline T stage & & & 0.866 \\
\hline $\mathrm{T} 1+\mathrm{T} 2$ & $9(9.4)$ & $12(7.8)$ & \\
\hline $\mathrm{T} 3$ & $76(79.2)$ & $121(79.1)$ & \\
\hline $\mathrm{T} 4$ & $11(11.5)$ & $20(13.1)$ & \\
\hline $\mathrm{N}$ stage & & & 0.976 \\
\hline No & $58(60.4)$ & $91(59.5)$ & \\
\hline $\mathrm{N} 1$ & $27(28.1)$ & $45(29.4)$ & \\
\hline $\mathrm{N} 2$ & $11(11.5)$ & $17(11.1)$ & \\
\hline TNM stage & & & 0.246 \\
\hline $\mathrm{IA}+\mathrm{IB}$ & $7(7.3)$ & $12(7.8)$ & \\
\hline IIA+IIB & $45(46.9)$ & $76(49.7)$ & \\
\hline IIIA+IIIB & $35(36.5)$ & $60(39.2)$ & \\
\hline IVA & $9(9.3)$ & $5(3.3)$ & \\
\hline
\end{tabular}

${ }^{a}$ Signet-ring cell carcinoma and mucinous adenocarcinoma. Values are expressed as n (\%) unless otherwise specified.

of PD-L1 in the tumor parenchyma distinguished the TCs and immune cells (ICs). For statistical analyses, the optimal cutoff value for each marker was determined using the minimum P-value, which was calibrated using the X-Tile tool (33). Each marker was stratified into dichotomous variables (high vs. low) and a calibrated KM analysis was recorded (Table SII).

Multiplex IHC (mIHC) and evaluation of staining. Measurement of CD8, CD33 and CK (separated tumor tissue) was performed by mIHC using the Opal iterative staining protocol according to the manufacturer's protocol (Akoya Biosciences) and previously published methods $(24,34)$. In brief, pretreatment of $\mathrm{mIHC}$ sections was performed as in the IHC assay described above and antigen retrieval was performed by microwave heating. Subsequently, sections were subjected to CD33, cytokeratin (CK) and CD8 Opal iterative staining and Opal 520, Opal 570 and Opal 620 (Akoya Biosciences) were applied to each antibody. Nuclei were counterstained with DAPI (Akoya Biosciences). The primary antibodies used for $\mathrm{mIHC}$ are listed in Table SI.

MIHC slide images were captured using the Vectra platform (Perkin-Elmer) and analyzed using inForm software version 2.2.1 (Akoya Biosciences). To better achieve the multispectral unmixing of mIHC fluorescence signals, the images of single stained slides of each marker were used to create a spectrum library and those of unstained slides were used to extract tissue autofluorescence. The quantification of positive cells for each marker was performed using the minimum region signal threshold in the inForm software automated counting tool. Measurements were recorded as the mean value 
of five randomly selected high-magnification (x200) fields on full slides. For CD8 ${ }^{+}$TIL, signals from tumor parenchyma (CK-positive) and mesenchyme (CK-negative) were counted. For CD $33^{+}$MDSCs, the levels were assessed in whole tumor regions (e.g., parenchyma and mesenchyme). Similarly, the minimum P-value of CD8 ${ }^{+}$TIL and CD $33^{+}$MDSC in mIHC was set as the optimal cutoff value.

Statistical analysis. Values are expressed as the mean \pm standard deviation. Statistical calculations were performed using SPSS Statistics (version 22; IBM Corporation) and GraphPad Prism (version 7; GraphPad Software, Inc.). The $\chi^{2}$ test was used to examine the categorized variables. For continuous variables, the Mann-Whitney U test was used. The correlation between the expression levels of each marker was determined using the Spearman coefficient and the correlation matrix was subjected to unsupervised hierarchical clustering. OS was defined as the time from surgical resection to last contact. The Kaplan-Meier method and log-rank test were used to evaluate OS. The univariate and multivariate Cox proportional hazards model was used to adjust the independent risk factors. Statistical significance was set at $\mathrm{P}<0.05$ (two-tailed).

Using the rms package in $\mathrm{R}$ version 3.6.1, a visualized nomogram based on the results of the Cox model analysis was developed using data from the primary cohort. The primary cohort served as the internal validation group for the nomogram, while external validation of the nomogram was performed using the validation cohort. The nomogram's model performance was quantified using Harrell's concordance index (C-index). Internal validation of the nomogram was performed using the bootstrap method and a relatively unbiased estimate was acquired through 1,000 repetitions. The predictive accuracy and recognition capacity of the nomogram using the internal and external validation cohorts was assessed by calculating the $\mathrm{C}$-index and calibration curve. To facilitate clinical practice, the nomogram was simplified and an immunoprofile based on the nomogram was constructed. Finally, the receiver operating characteristic (ROC) curve of the immunoprofile and TNM stage system were compared in patients with stage II/III CRC.

\section{Results}

Patient clinical characteristics in both cohorts. The basic clinical and pathological features of the patients with CRC from the two cohorts are listed in Table I. No significant differences in clinical characteristics between the two cohorts were observed (Table I). At the time of diagnosis, in the primary cohort, the median age was 62.5 (range, 24-90) years, 63 (65.6\%) patients were male, and in the validation cohort, the median age was 66 (range, 22-95) years, of which 89 (58.2\%) patients were male. In the present study, most patients presented with locally advanced stages of the disease (TNM stage II and III), accounting for $83.3 \%(80 / 96)$ in the primary cohort and $88.9 \%(136 / 153)$ in the validation cohort. Postoperative adjuvant therapy was as follows: Patients with TNM stage I entered the follow-up period directly after curative surgical resection, whereas most patients with TNM stage II/III received close surveillance and capecitabine or 5-FU-based standard adjuvant chemotherapy. Advanced patients were given systemic treatment. The patients of the two cohorts were followed up for at least 5 years.

\section{CRC immune markers}

Infiltrating ICs. Infiltration of CD4 $4^{+}$TILs, CD $8^{+}$TILs, Foxp $3^{+}$Tregs and CD $33^{+}$MDSCs at the CRC tumor in situ was observed (Fig. 1, upper panel). In CRC, the location and density of the four tumor-infiltrating ICs were heterogeneous. As presented in Fig. 1, the compositions of CD4 ${ }^{+}$TILs, CD8 ${ }^{+}$TILs and Foxp3 ${ }^{+}$Tregs were different in the tumor parenchyma and mesenchyme regions. These ICs, particularly $\mathrm{CD} 4^{+} \mathrm{TIL}$ and $\mathrm{CD} 8^{+} \mathrm{TIL}$, were markedly higher in the tumor mesenchyme than in the tumor parenchyma. Immunopositives in the tumor parenchyma and mesenchyme were described as '(P)' and '(M)', respectively. In the primary cohort, $85 \%$ of patients (82/96; P) and $97 \%$ of patients $(94 / 96$; M) had at least $1 \% \mathrm{CD}^{+} \mathrm{TIL}$ infiltration. A total of $26 \%$ of patients (25/96; P) and $100 \%$ of patients $(96 / 96 ; \mathrm{M})$ had at least $1 \% \mathrm{CD} 4^{+} \mathrm{TIL}$ infiltration. Furthermore, $21 \%$ of patients (20/96; P) and $97 \%$ of patients $(94 / 96 ; \mathrm{M})$ had at least $1 \%$ Foxp3 ${ }^{+}$Treg infiltration. Furthermore, the infiltration of $\mathrm{CD} 33^{+} \mathrm{MDSC}$ was relatively sparse in the tumor mesenchyme and parenchyma. Thus, the analysis of $\mathrm{CD} 33^{+} \mathrm{MDSC}$ was performed in the entire tumor region and at least $1 \% \mathrm{CD} 33^{+} \mathrm{MDSC}$ infiltration was noted in $48 \%$ of patients (46/96) (Table SII).

Expression of co-signaling molecules and immunomodulatory factors. The expression levels of PD-L1, PD1, LAG3, TIM3, OX40, ICOS and IDO1 were examined in the CRC tumors in situ by IHC (Fig. 1, middle and lower panels). Overall, the expression rate of PD-L1 in tumor parenchyma was low, with only seven patients (7/96) expressing $\geq 1 \%$ PD-L1 in TCs, and only one case (1/7) with 5\% PD-L1 in ICs, whereas $70 \%$ (67/96) of the patients had at least 1\% PD-L1 expression in the tumor mesenchyme. At least 1\% PD-1 expression was observed in $21 \%$ of patients $(20 / 96$; P) and $60 \%$ of patients $(58 / 96 ; \mathrm{M})$. The positive expression was defined as $\geq 1 \%$ expression in ICs. The expression of LAG3, TIM3, OX40 and ICOS was $8 \%(8 / 96), 43 \%$ (41/96), 59\% (57/96) and 79\% (76/96), respectively. Furthermore, IDO1 was observed in both TCs and ICs. When considering $\geq 1 \%$ to indicate positive staining, it was observed that $36 \%$ of patients (35/96) exhibited IDO1 expression in TCs, while $89 \%$ of patients (85/96) expressed IDO1 in ICs.

Correlation between tumor-infiltrating ICs and co-signaling molecules or immunomodulatory factors. According to the mechanisms governing tumor immunology, ICs may be regulated by the expression of activated and inhibited molecules, which may shift the immune response toward anti-inflammatory profiles or escaped antitumor immunity. Thus, the correlation between CD8 ${ }^{+}$TIL, CD $4^{+} \mathrm{TIL}$, Foxp3 ${ }^{+}$Treg, $\mathrm{CD}_{3} 3^{+} \mathrm{MDSC}$ and PD-L1, PD-1, LAG3, TIM3, OX40, ICOS and IDO1 was analyzed (Fig. S1). The densities of CD8 ${ }^{+} \mathrm{TIL}$, both in the tumor parenchyma and mesenchyme, were significantly correlated with the expression of PD-L1 (P), PD1 (M), TIM3, OX40 and ICOS (all P<0.001) and increased along with CD4 ${ }^{+}$TIL (M) and Foxp3 ${ }^{+}$Treg (M) (all $\mathrm{P}<0.001$ ). By contrast, $\mathrm{CD} 33^{+} \mathrm{MDSC}$ were associated with the infiltration of $\mathrm{CD} 4^{+} \mathrm{TIL}(\mathrm{P}<0.001)$ and expression of TIM3 $(\mathrm{P}=0.016)$. In addition, CD4 ${ }^{+}$TIL $(\mathrm{M})$ and Foxp $3^{+}$Treg $(\mathrm{M})$ were also 


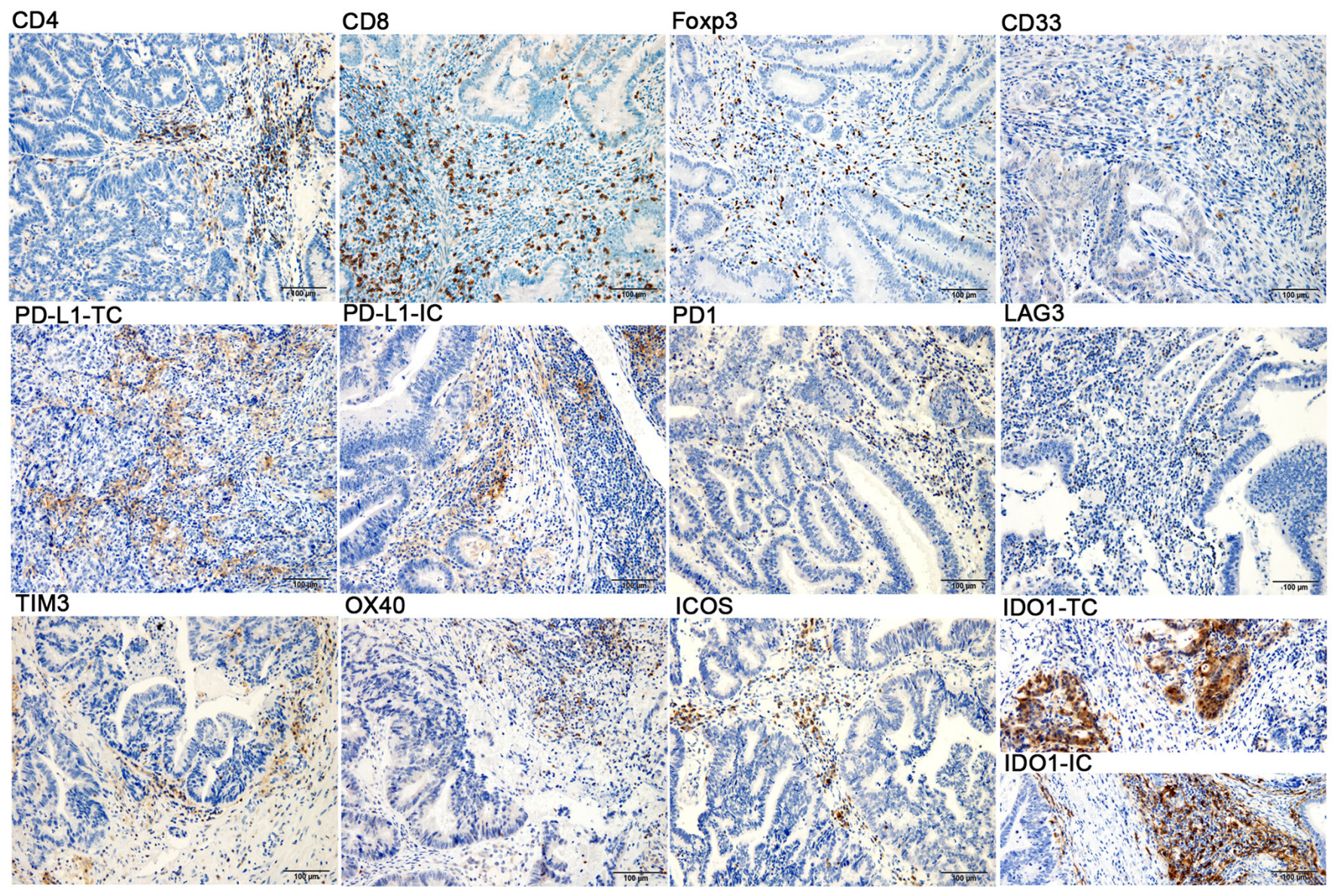

Figure 1. Immunohistochemical staining of CD4, CD8, Foxp3, CD33, PD-L1, PD1, LAG3, TIM3, OX40, ICOS and IDO1 in colorectal cancer samples (magnification, x200; scale bar, $100 \mu \mathrm{m}$; blue, nuclear staining; brown, positive staining). TC, tumor cell; IC, immune cell; Foxp3, forkhead box p3; PD1, programmed cell death 1; PD-L1, PD1 ligand 1; TIM3, T-cell immunoglobulin mucin family member 3; LAG3, lymphocyte-activating 3; OX40, tumor necrosis factor receptor superfamily, member 4; ICOS, inducible T-cell costimulator; IDO1, indoleamine 2,3-dioxygenase 1.

positively correlated with the expression of PD-L1 (P), PD1 (M), TIM3, OX40, ICOS and IDO1 (all P<0.001). These correlations may reflect a highly activated immune microenvironment in CRC.

Association between immune markers and clinical outcomes Infiltrating ICs and clinical outcomes. The relationship between the patients' OS and CD4 ${ }^{+}$TIL, CD8 $8^{+}$TIL and Foxp $3^{+}$Treg expression in the tumor parenchyma and mesenchyme was evaluated. The survival curves indicated that the favorable prognosis of patients with high densities of CD8 ${ }^{+} \mathrm{TIL}$, both in the tumor parenchyma and mesenchyme according to IHC and mIHC staining, was statistically significant compared to those with low CD8 ${ }^{+}$TIL (Fig. 2A and B). The densities of CD8 ${ }^{+}$TIL in both tumor regions were analyzed in combination, with poor infiltration defined as $\leq 1 \%$ CD8 $8^{+}$TIL in the parenchyma, together with $\leq 5 \% \mathrm{CD} 8^{+}$TIL in the mesenchyme, whereas other combinations were regarded as rich infiltration (Figs. 2B and S2). In both cohorts, the CD8+TIL rich groups were significantly associated with prolonged patient survival $(\mathrm{P}=0.0002$ in the primary cohort and $\mathrm{P}<0.001$ in the validation cohort; Fig. 2B). The favorable prognostic effect mentioned above was not observed in the parenchyma, mesenchyme or whole tumor region for either CD4 $4^{+}$TIL or Foxp3 ${ }^{+}$Tregs (Fig. S3A). Of note, the analysis of $\mathrm{CD}^{+} 3^{+} \mathrm{MDSC}$ revealed that a high density of $\mathrm{CD} 33^{+} \mathrm{MDSC}$ resulted in a significantly unfavorable prognosis compared to low $\mathrm{CD} 33^{+} \mathrm{MDSC}$ in both cohorts of patients with $\mathrm{CRC}(\mathrm{P}=0.0019$ in the primary cohort and $\mathrm{P}=0.0003$ in the validation cohort; Fig. $2 \mathrm{C}$ and $\mathrm{D})$.

Next, the prognostic value of the different proportions of the $\mathrm{CD} 8^{+}$TILs and CD33 ${ }^{+}$MDSCs when they appeared simultaneously in the CRC microenvironment was analyzed (Fig. 2E and F). Tumors with rich CD8 ${ }^{+}$TILs in the TME (TME-activated) were associated with favorable survival, whereas tumors with high $\mathrm{CD} 33^{+} \mathrm{MDSC}$ (TME-inhibitory) were associated with poor clinical outcomes. Near-equal infiltration of $\mathrm{CD}^{+} \mathrm{TIL}$ and $\mathrm{CD} 33^{+}$MDSCs tended to indicate an equilibrium TME, in which the patients had inferior survival compared to those with an activated TME. However, no significant differences were observed between TME-equilibrium and TME-inhibitory in the validation cohort. These data highlight the importance of defining a good scoring system to assess the status of TME in CRC.

Co-signaling molecules or immunomodulatory factors and clinical outcomes. Among the six co-signaling molecules examined, only PD1 (P) and PD1 (M) were significantly associated with favorable $\mathrm{OS}(\mathrm{P}=0.0421$ and $\mathrm{P}=0.0197$, respectively), whereas PD-L1 (P), PD-L1 (M), LAG3, TIM3, OX40, ICOS, IDO1 (P) and IDO1 (M) were not significantly associated with any outcome (Fig. S3B and C). To further assess 
A

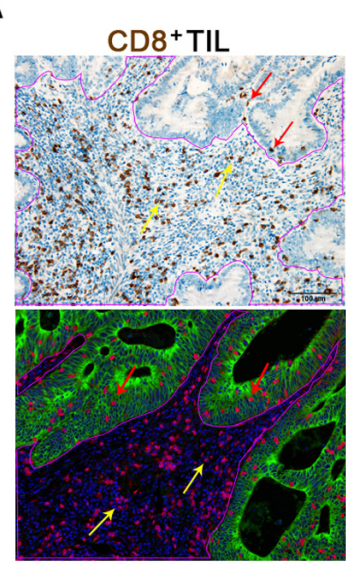

CD8/CK/DAPI

C

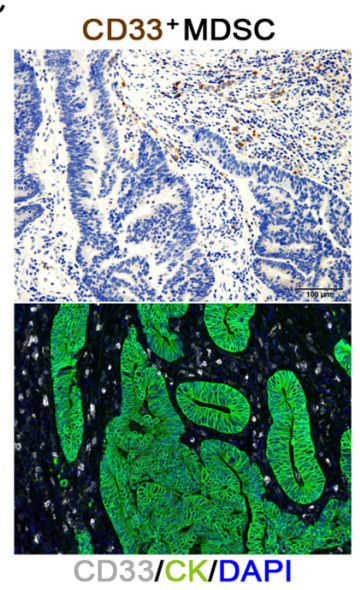

E
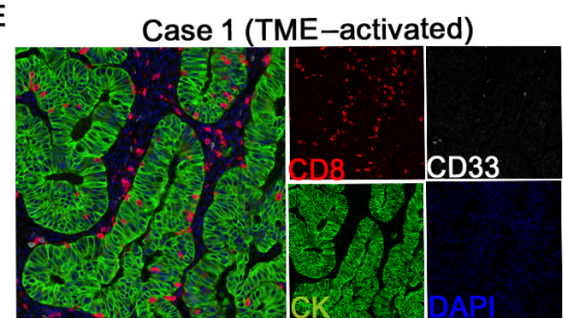

F

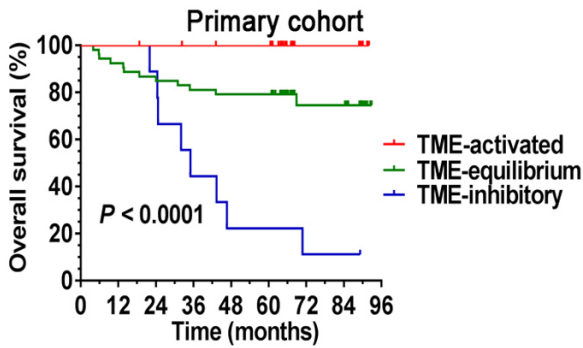

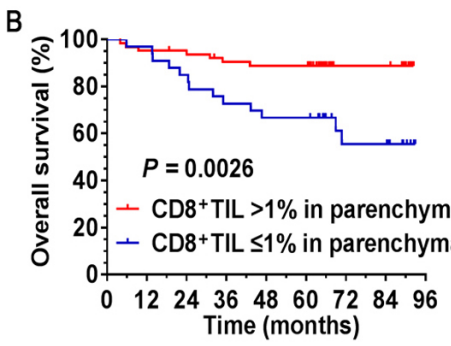
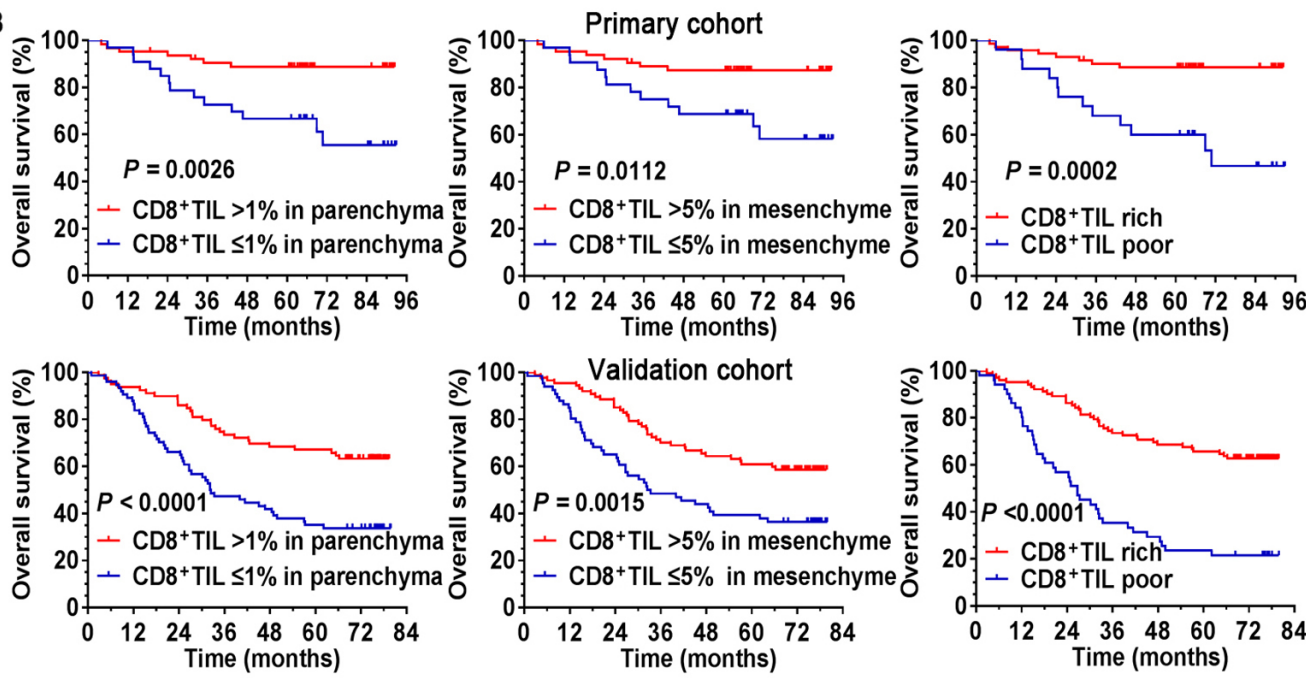

Primary cohort
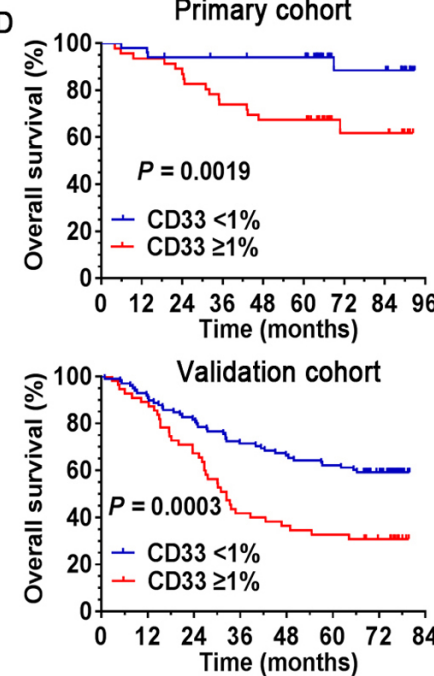

Time (months)
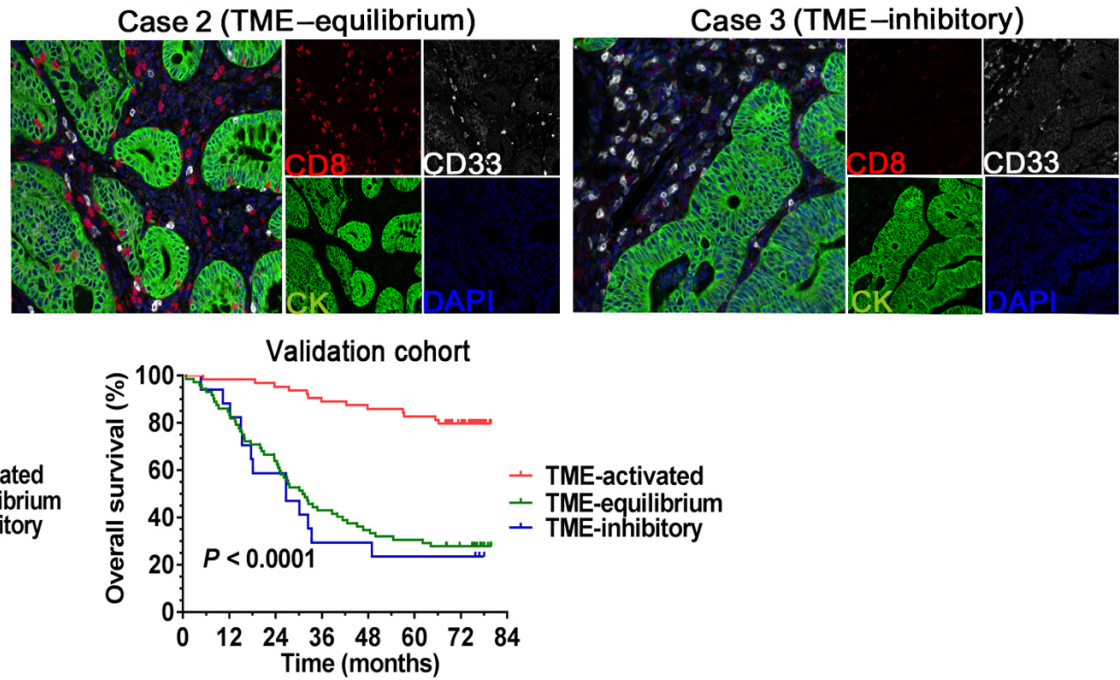

Figure 2. Association between the immune cells and clinical outcomes. (A) IHC and mIHC staining of CD8 ${ }^{+}$TIL in the tumor parenchyma (red arrow) and mesenchyme (yellow arrow), and the solid purple line is the delimitation of parenchyma/mesenchyme. (B) Survival curves comparing OS of patients stratified by different CD8 ${ }^{+}$TIL infiltration status. (C) IHC and mIHC staining of CD33 ${ }^{+}$MDSCs. The assessment of CD33 ${ }^{+}$MDSCs was performed in the entire tumor region. (D) Survival curves comparing OS of patients stratified by different CD $33^{+}$MDSC infiltration status. (E) Representative mIHC images for three different statuses of tumor-infiltrating immune cells in the TME (magnification, x200; scale bar, $100 \mu \mathrm{m}$ ). (F) Survival curves comparing the OS of patients stratified by different TME status. MDSCs, myeloid-derived suppressor cells; OS, overall survival; IHC, immunohistochemistry; mIHC, multiplex IHC; TME, tumor microenvironment; TIL, tumor-infiltrating lymphocytes; CK, cytokeratin.

the prognostic significance of PD1, PD1 (P) and PD1 (M) were defined, with $\geq 1 \%$ as $P D 1^{+}$TIL-rich and the others as
PD1+TIL-poor. Similarly, PD1+TIL-rich cells were significantly associated with prolonged patient survival $(\mathrm{P}=0.0459$; 
Table II. Univariate/multivariate analyses for overall survival in the primary cohort.

\begin{tabular}{|c|c|c|c|c|}
\hline \multirow[b]{2}{*}{ Prognostic factor } & \multicolumn{2}{|c|}{ Univariate analyses } & \multicolumn{2}{|c|}{ Multivariate analyses } \\
\hline & Hazard ratio & P-value & Hazard ratio & P-value \\
\hline Age, $<63$ vs. $\geq 63$ years & $1.596(0.652-3.907)$ & 0.306 & & \\
\hline Male vs. female sex & $0.465(0.155-1.392)$ & 0.171 & & \\
\hline History of smoking, yes vs. no & $1.807(0.752-4.344)$ & 0.186 & & \\
\hline History of drinking, no vs. yes & $1.517(0.551-4.178)$ & 0.420 & & \\
\hline $\begin{array}{l}\text { Histological type, } \\
\text { adenocarcinoma vs. other }{ }^{\mathrm{a}}\end{array}$ & $2.643(0.351-19.889)$ & 0.345 & & \\
\hline Vascular invasion, no vs. yes & $2.578(0.754-8.820)$ & 0.131 & & \\
\hline Grade, G1 vs. G3 & $0(0)$ & 0.983 & & \\
\hline Grade, G2 vs. G3 & $0.613(0.223-1.688)$ & 0.344 & & \\
\hline $\mathrm{T}$ stage, $\mathrm{T} 1+\mathrm{T} 2$ vs. $\mathrm{T} 4$ & $0(0)$ & 0.980 & & \\
\hline T stage, $\mathrm{T} 3$ vs. T4 & $0.304(0.116-0.796)$ & 0.015 & & \\
\hline $\mathrm{N}$ stage, $\mathrm{N} 0$ vs. $\mathrm{N} 2$ & $0.193(0.061-0.609)$ & 0.005 & & \\
\hline $\mathrm{N}$ stage, $\mathrm{N} 1$ vs. $\mathrm{N} 2$ & $0.439(0.138-1.396)$ & 0.163 & & \\
\hline AJCC TNM stage, I vs. IV & $0(0)$ & 0.976 & $0(0)$ & 0.983 \\
\hline AJCC TNM stage, II vs. IV & $0.080(0.023-0.279)$ & $<0.001$ & $0.129(0.034-0.498)$ & 0.003 \\
\hline AJCC TNM stage, III vs. IV & $0.248(0.086-0.712)$ & 0.010 & $0.358(0.119-1.077)$ & 0.068 \\
\hline CD4+TIL, poor vs. rich & $1.206(0.495-2.941)$ & 0.680 & & \\
\hline $\mathrm{CD} 8+\mathrm{TIL}$, poor vs. rich & $0.216(0.088-0.530)$ & 0.001 & $0.201(0.056-0.727)$ & 0.014 \\
\hline Foxp3+TIL, poor vs. rich & $1.336(0.546-3.271)$ & 0.525 & & \\
\hline $\mathrm{CD}^{2} 3^{+} \mathrm{MDSC}$, low vs. high & $4.816(1.609-14.414)$ & 0.005 & $4.565(1.428-14.592)$ & 0.010 \\
\hline PD-L1-parenchyma, low vs. high & $1.618(0.374-6.998)$ & 0.520 & & \\
\hline PD-L1-mesenchyme, low vs. high & $0.454(0.188-1.098)$ & 0.080 & $0.631(0.222-1.792)$ & 0.388 \\
\hline PD1 $1^{+} \mathrm{TIL}$, poor vs. rich & $0.418(0.173-1.011)$ & 0.053 & $0.796(0.228-2.776)$ & 0.720 \\
\hline LAG3, low vs. high & $0.042(0.000-27.922)$ & 0.340 & & \\
\hline TIM3, low vs. high & $0.903(0.369-2.210)$ & 0.823 & & \\
\hline OX40, low vs. high & $0.525(0.217-1.269)$ & 0.152 & & \\
\hline ICOS, low vs. high & $0.427(0.174-1.045)$ & 0.062 & $1.407(0.392-5.054)$ & 0.600 \\
\hline IDO1-parenchyma, low vs. high & $1.735(0.709-4.249)$ & 0.228 & & \\
\hline IDO1-mesenchyme, low vs. high & $0.622(0.226-1.716)$ & 0.359 & & \\
\hline
\end{tabular}

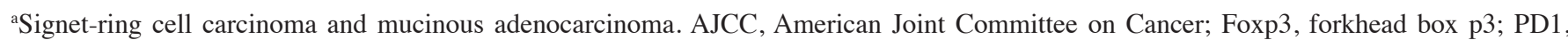
programmed cell death 1; PD-L1, PD1 ligand 1; TIM3, T-cell immunoglobulin mucin family member 3; LAG3, lymphocyte-activating 3; OX40, tumor necrosis factor receptor superfamily, member 4; ICOS, inducible T-cell costimulator; IDO1, indoleamine 2,3-dioxygenase 1.

Fig. S3B). IDO1 was observed to be expressed by TCs and ICs (Fig. 1, lower panels) and Kaplan-Meier survival analyses suggested that no statistically significant differences were present between groups with high and low IDO1 expression levels in the different cells (Fig. S3C).

Construction and validation of the nomogram-based immunoprofile

Independent prognostic factors. First, the hazard ratio of the clinical parameters and immune markers was analyzed in the primary cohort using a univariate regression model. As presented in Table II, among the clinical characteristics, only pathologic T stage, $\mathrm{N}$ stage and TNM stage were associated with increased risk of death and OS (Fig. S4A-C). Subsequently, TNM stage and immunological markers with $\mathrm{P}<0.1$ in the univariate analysis were incorporated into a Cox proportional regression model for multivariate analysis (Table II). It was determined that $\mathrm{CD} 8(\mathrm{HR}=0.201 ; 95 \% \mathrm{CI}: 0.056-0.727$; $\mathrm{P}=0.014), \mathrm{CD} 33$ ( $\mathrm{HR}=4.565 ; 95 \% \mathrm{CI}: 1.428-14.592 ; \mathrm{P}=0.010)$ and TNM stage (II vs. IV, HR=0.129; $95 \%$ CI: 0.034-0.498; $\mathrm{P}=0.003$ ) exhibited independent prognostic value for OS in patients with CRC.

Construction of the nomogram-based immunoprofile and validation. Although both CD8 ${ }^{+} \mathrm{TIL}$ and $\mathrm{CD} 33^{+} \mathrm{MDSC}$ had independent prognostic significance in the multivariate analyses (Table II), they were reported to have functionally opposite effects during the antitumor immune responses in patients with CRC $(11,35)$, preventing their use for accurate prediction of survival. Therefore, a comprehensive immunoprofile nomogram for predicting survival was created using the independent prognostic variables (Fig. 3A). The C-index for the predicted OS was 0.861 (95\% CI: 0.796-0.925) in the internal validation and 0.759 (95\% CI: 0.714-0.804) in the external validation cohort. The calibration plot for the 
A

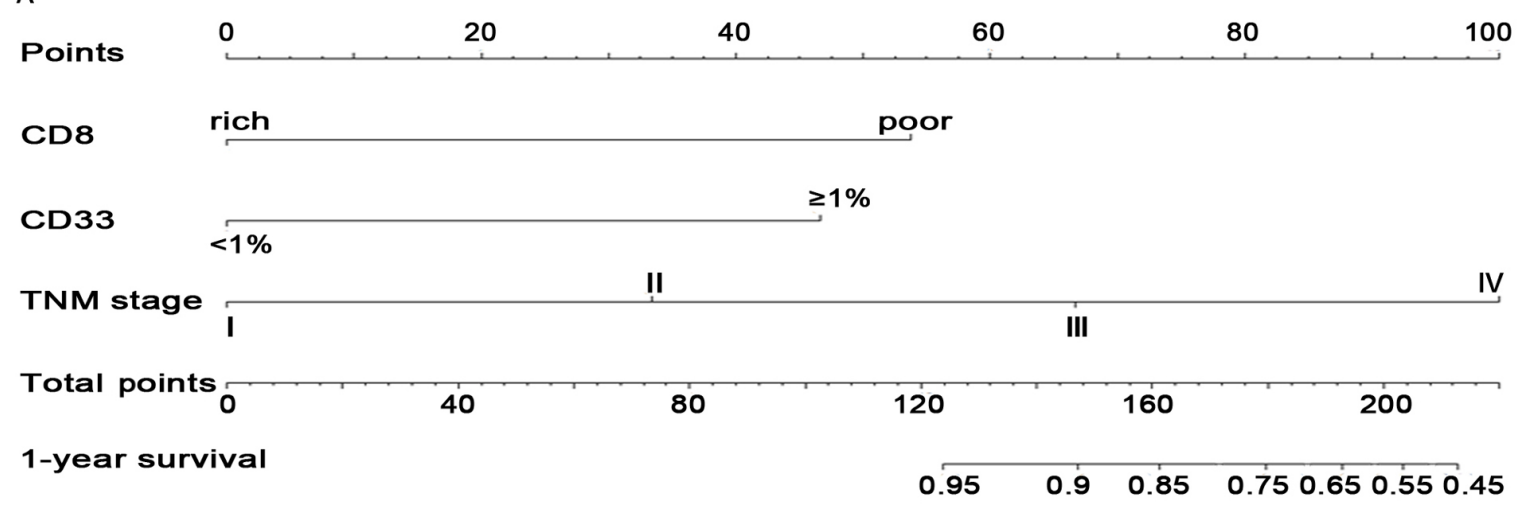

\section{2-year survival}

$\begin{array}{llllllllllllllll}0.9 & 0.8 & 0.7 & 0.6 & 0.5 & 0.4 & 0.3 & 0.2 & 0.1\end{array}$

\section{3-year survival}

B

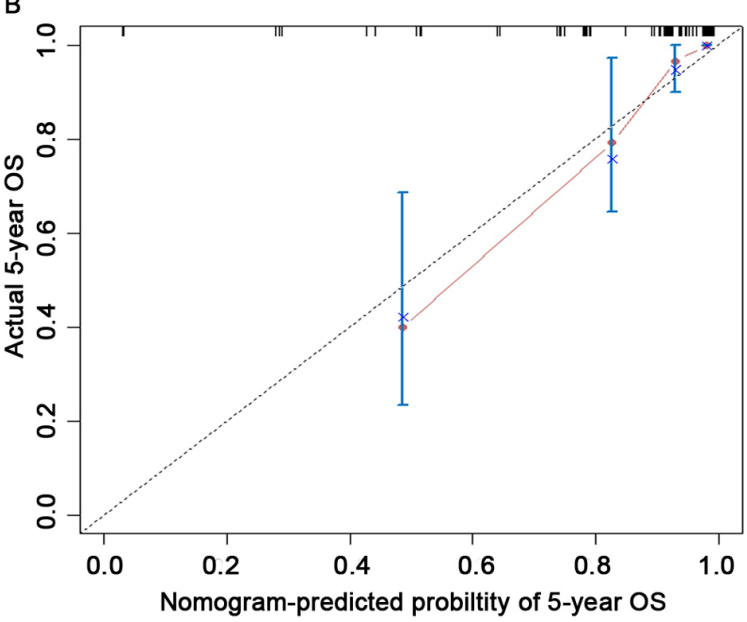

0.9
C

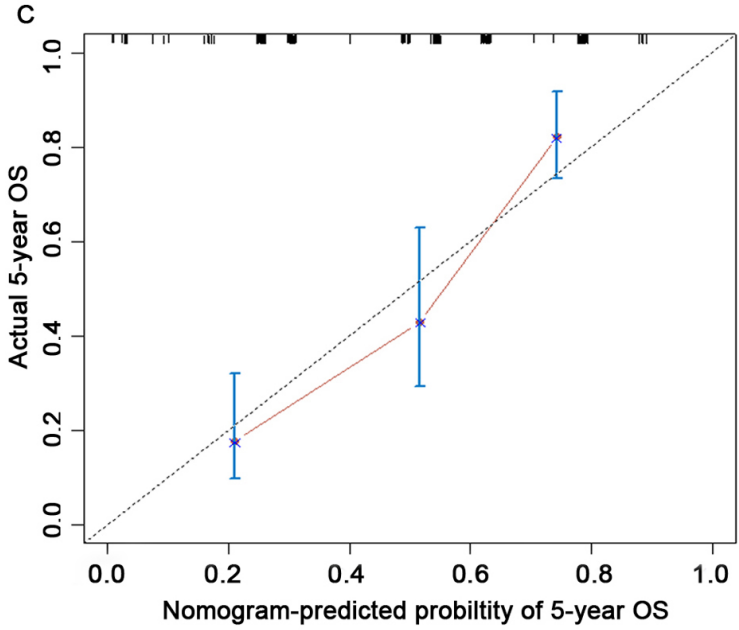

Figure 3. Construction and validation of the prognostic nomogram. (A) The nomogram was constructed using independent prognostic variables of the multivariate analysis in patients with colorectal cancer. (B and C) Calibration curves of the nomogram for predicting 5-year patient survival in (B) the primary cohort and (C) the validation cohort. OS, overall survival.

probability of 5-year OS between the actual observed survival and the prediction by the nomogram was in good accordance in the primary cohort (Fig. 3B) as well as in the validation cohort (Fig. 3C).

To facilitate clinical application, a recent study by our group proposed modifying a nomogram into a simple immunoprofile system based on a nomogram for patients with esophageal cancer (24). In the present study, an immunoprofile for patients with CRC was developed. In the immunoprofile system, 0, 1, 2 and 3 points corresponded to stages I, II, III and IV, respectively. The points for CD8-poor and $\mathrm{CD} 33^{+} \mathrm{MDSC} \geq 1 \%$ were between stage II and III in the nomogram; thus, 1.5 points was assigned in the immunoprofile. Next, each patient was assigned an immunoprofile index by adding the values of CD8 and CD33. Patients with different immunoprofile indices had variant clinical outcomes: Patients with a high immunoprofile index had unfavorable OS; patients with the lowest immunoprofile index had the highest OS; other patients had intermediate clinical outcomes (Fig. S4D).

Comparison of the predictive accuracy for OS between the immunoprofile and TNM staging systems. The ability of the immunoprofile to identify differences in patients at the same
TNM stage was further investigated. As presented in Fig. 4A, all patients $(n=249)$ were divided into two risk subgroups based on their immunoprofile for CD8 ${ }^{+}$TIL and CD $33^{+}$MDSCs, regardless of TNM stage, using a value of 1.5 as the cutoff. During the observation period, $86.7 \%$ of patients with an immunoprofile index $<1.5$ were still alive and only $43.7 \%$ of cases were still alive in the high immunoprofile index $(\geq 1.5)$ group. The immunoprofile system was able to separate patients at the same tumor stage into different risk subgroups (Fig. 4B-E), particularly for TNM stages II $(\mathrm{P}<0.0001)$ and III $(\mathrm{P}=0.0002)$, where significant separation was noted in these patients.

For the patients at stage II/III $(n=216)$, the predictive accuracy for OS between the immunoprofile and TNM stage system was compared by ROC curve analysis. The area under the ROC curve (AUC) of the immunoprofile and TNM staging system was 0.702 (95\% CI: 0.636-0.762) and 0.613 (95\% CI: 0.0544-0.0678), respectively (Fig. 4F). The Z-statistic value of the pairwise comparison of ROC curves was 1.995, exhibiting a statistically significant difference $(\mathrm{P}=0.046)$. These results demonstrated that the immunoprofile provided a more accurate prognosis for patients with stage II/III CRC compared with the TNM staging system. 

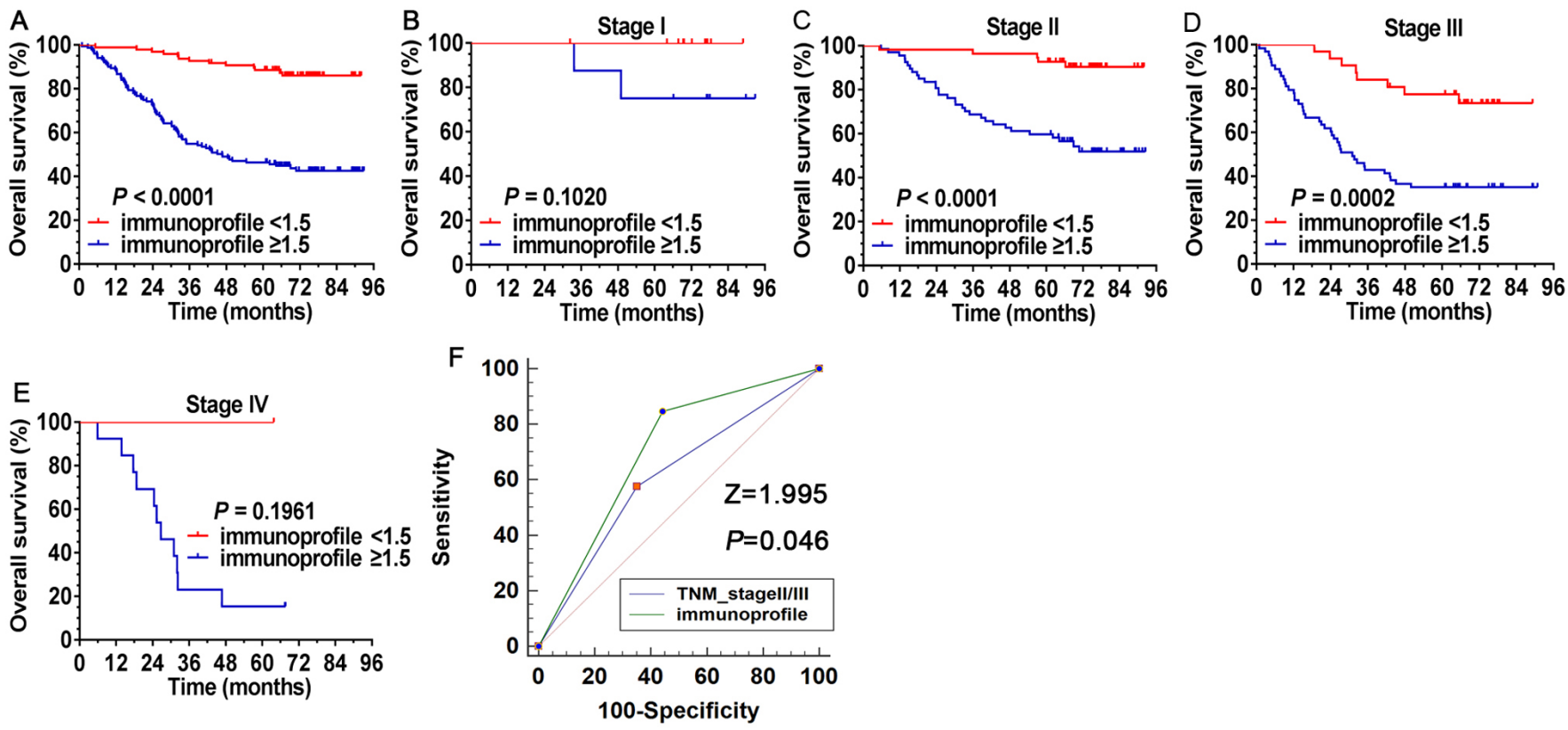

Figure 4. Survival analysis of the immunoprofile based on $\mathrm{CD}^{+}$tumor-infiltrating lymphocytes and CD33 ${ }^{+}$myeloid-derived suppressor cells. (A) Based on the immunoprofile, all patients $(\mathrm{n}=249)$ were divided into different risk subgroups, regardless of TNM stage, with a cutoff value of 1.5. (B-E) The immunoprofile separated contemporaneous patients into different risk subgroups, particularly for patients with TNM stage II/III, who exhibited a significant separation. (B) TNM stage I, (C) TNM stage II, (D) TNM stage III and (E) TNM stage IV. (F) ROC curves were compared between the immunoprofile and the TNM stage system in patients with stage II/III colorectal cancer $(n=216)$. The Z-statistic value of the pairwise comparison of the ROC curves was 1.995 , with a statistically significant difference $(\mathrm{P}=0.046)$. ROC, receiver operating characteristic.

\section{Discussion}

Cancer has traditionally been defined with a cellular-centered vision, but this is being replaced by a holistic vision that includes the microenvironment (36). The immune microenvironment has an important role in the evolution of tumors (37), and the immune components within the microenvironment have a crucial impact on the clinical outcomes for tumor patients (8). $\mathrm{CRC}$ is a chronic mucosal inflammatory-related malignancy (2). Pagès et al (13) first established an immunoscore in CRC that included only CD3/CD8 adaptive lymphocytes and did not consider immunosuppressive markers. In the present study, to improve the application of immune parameters in the prognosis assessment of patients, for the first time, the density and location of 11 immunological markers in situ was simultaneously analyzed and an immune-related prognostic nomogram for patients with CRC was constructed. To facilitate routine clinical usage and discriminate the nomogram system from the previous immunoscore algorithm, the system of the present study was modified and defined as an 'immunoprofile'. The present results indicated that the immunoprofile system had a good predictive performance. The AUC for predicting OS in patients with stage II/III was 0.702 , significantly better than that for the TNM II/III stage $(\mathrm{AUC}=0.613$ ) (Z-value $=1.995, \mathrm{P}=0.046$ ).

The present immunoprofile model utilized tumor-infiltrating CD8 ${ }^{+}$TIL and $\mathrm{CD}_{3} 3^{+} \mathrm{MDSC}$, which are significantly associated with clinical outcomes for CRC. CD8 ${ }^{+} \mathrm{T}$ lymphocytes are the most important immune effector and have a central role in eliminating TCs (38). The prognostic role of CD8 ${ }^{+} \mathrm{TIL}$ as the most robust immune biomarker has been reported in various cancer types $(8,17)$. The results of the present study are consistent with those of previous reports $(11,39)$ in terms of high CD8 ${ }^{+}$TIL infiltration being associated with improved patient survival and being an independent prognostic factor according to multivariate analyses. Furthermore, the distribution of $\mathrm{CD}^{+}{ }^{+}$TILs in the human tumor regions was not random, which corroborated reports that infiltration to specific regions varies according to the different tumor types $(6,40,41)$. In the present study, it was observed that the increased density of CD8-TILs in the parenchyma was more essential for the prognosis of CRC. Compared with CD8 ${ }^{+}$TILs infiltrating the mesenchyme, $\mathrm{CD} 8^{+}$TILs in the parenchyma exerted a greater antitumor immune response. Correlation analysis suggested that infiltration of CD8 ${ }^{+}$TIL was significantly correlated with the expression of PD-L1, PD-1, TIM3, OX40 and ICOS. The PD-1/PD-L1 pathway has an important role in inhibiting the immune response and controls the induction and maintenance of immune tolerance in the tumor microenvironment (42). TIM3, another inhibitive immune checkpoint molecule, is expressed in $\mathrm{CD}^{+} \mathrm{T}$ cells and is thought to be involved in T-cell differentiation and activation, and its persistence may be associated with an exhaustion status (43). OX40 is a co-stimulatory molecule, which, if in contact with the OX40 ligand expressed by antigen-presenting cells, promotes the proliferation of $\mathrm{CD}^{+}$and $\mathrm{CD} 8^{+} \mathrm{T}$ cells and the survival of antigen-specific memory $\mathrm{T}$ cells (44). Evidence suggests that OX40 expression improves the prognostic significance of CD8 ${ }^{+}$T-cell infiltration in CRC (45). ICOS is another T-cell co-stimulatory molecule. ICOS is expressed on activated $\mathrm{T}$ cells, memory $\mathrm{T}$ cells and regulatory $\mathrm{T}$ cells. Similarly, ICOS binds to its ligand (ICOSL), which regulates T-cell proliferation and survival, as well as stimulates the production of cytokines (46). Thus, these T-cell inhibitory and activating 
receptors modulate the balance between immune tolerance and immune response in the CRC tumor (29). However, these co-inhibitors and co-stimulators are expressed simultaneously or at different times on $\mathrm{CD}^{+} \mathrm{T}$ cells, resulting in different phenotypes (47). The relationship between these phenotypes and the prognosis of patients with CRC remains to be fully elucidated and requires to be further investigated. In addition, there is growing evidence that $\mathrm{CD}^{+}{ }^{+} \mathrm{T}$ cells have a vital role in anti-tumor immunity, specifically designed to activate the $\mathrm{CD}^{+}$cytotoxic T-lymphocyte response (48). The present correlation analysis indicated that the infiltration of CD8 $8^{+}$TIL was associated with increased CD4 ${ }^{+}$TIL, suggesting that $\mathrm{CD} 4^{+}$TIL have an adjunct role in the efficacy of anti-tumor $\mathrm{CD}^{+} \mathrm{TIL}$ responses in the CRC microenvironment. Furthermore, correlation analysis also revealed that the infiltration of $\mathrm{CD} 8^{+}$TIL was associated with increased Foxp $3^{+}$Tregs, which may also demonstrate the negative feedback mechanism of the immune system. However, this hypothesis requires to be further analyzed and verified by Digital Spatial Profiling (49).

MDSCs represent a heterogeneous population of pathologically activated immature myeloid cells that have potent immune suppressive activity in peripheral lymphoid organs and tumor in situ, and the abnormal accumulation of these cells is an important mechanism for tumor immune evasion $(22,50)$. Previous studies have indicated that the proportion of circu-

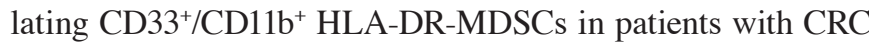
was higher than that in healthy individuals and the percentage of MDSCs in the peripheral blood of patients with CRC was closely correlated with clinical cancer stage and distant metastasis $(35,51)$. Furthermore, recent evidence has revealed that the expansion of circulating granulocytic MDSCs was associated with poor prognosis in patients with metastatic CRC treated with FOLFOX-Bevacizumab chemotherapy (52). Although tumor tissue was included in certain studies $(35,51)$, the significance was limited by low sample numbers. The present study first analyzed the relationship between the infiltration of $\mathrm{CD} 33^{+} \mathrm{MDSC}$ in situ and the risk of death in patients with CRC. It was revealed that a high density of CD $33^{+} \mathrm{MDSCs}$ in the tumor in situ was associated with poor outcome compared with a low density of CD $33^{+}$MDSCs. Multivariate analyses suggested that $\mathrm{CD} 33^{+} \mathrm{MDSC}$ were an independent prognostic factor in CRC. Furthermore, it was observed that tumor-infiltrating $\mathrm{CD}_{3} 3^{+} \mathrm{MDSCs}$ were closely correlated with the infiltration of CD4+TILs $(\mathrm{P}<0.001)$, suggesting that MDSCs affect antitumor immunity by $\mathrm{CD} 4^{+} \mathrm{T}$ cells in $\mathrm{CRC}$ in situ (53); however, further investigation is required to confirm their immunosuppressive function.

In the tumor microenvironment, different ICs lead to different TME statuses and affect the prognosis of patients. Based on immune effector CD8 ${ }^{+}$TIL and immunosuppressive CD $33^{+} \mathrm{MDSC}$, the present immunoprofile system demonstrated good predictive performance for patients with stage II/III CRC. However, the present study also has several limitations. First, the sample size of stage I/IV patients was too small, limiting the performance evaluation of the immunoprofile system, which thus requires further research. In addition, the immune factors used in the present study were quite limited. With the development of cancer and immune microenvironment research, there is no doubt that continuous improvement of the immunoprofile will be implemented, incorporating more prognostic parameters and improving the predictive model. Furthermore, the prognostic value of tumor-infiltrating Foxp $3^{+}$Tregs in CRC remains controversial. Certain studies have indicated that Foxp $3^{+}$Tregs are associated with favorable prognosis in CRC, whereas the results of the present study did not indicate such positive prognostic value. Therefore, future investigations should distinguish Treg-cell subpopulations (21) or define a suppressive index of Treg cells (40) in CRC, and to explore the underlying mechanisms governing the roles of these cells.

In conclusion, in the present study, a nomogram-based immunoprofile system based on CD8 ${ }^{+}$TILs and CD $33^{+}$MDSCs was established in patients with CRC. The results demonstrated that the immunoprofile provides accurate prognosis prediction and is an important supplement to the TNM staging system for patients with stage II/III CRC.

\section{Acknowledgements}

The authors would like to acknowledge Dr Lai Song from Beijing DCTY Bio-information Technology Co. (Beijing, China) for excellent technical assistance in data analysis.

\section{Funding}

This study was supported by the National Natural Science Foundation of China (grant no. 81672274) and the Clinical and Scientific Research Support Fund of the General Hospital of the Chinese PLA (grant no. 2017-FC-TSYS-3022).

\section{Availability of data and materials}

The datasets used and/or analyzed during the current study are available from the corresponding author upon reasonable request.

\section{Authors' contributions}

LXW, SCJ, QW and JQH contributed to the conception and design of the study. LXW, NJC and JFL collected the data. LXW, NJC, ZZ and LJZ performed IHC and mIHC staining. LXW, LLW and YC performed the statistical analysis and interpretation. LXW was a major contributor in writing the manuscript. All authors contributed to critical revision of the final manuscript and approved the manuscript for submission. LXW and SCJ confirm the authenticity of the raw data.

\section{Ethics approval and consent to participate}

In this study, the Chinese PLA General Hospital was authorized to use the cohort of Qingyang People's Hospital. The study was approved by the Ethics Committee of the Chinese PLA General Hospital (Beijing, China; approval no. S2019-228-02) and written informed consent was provided by all of the patients.

\section{Patient consent for publication}

Not applicable. 


\section{Competing interests}

The authors declare that they have no competing interests.

\section{References}

1. Wittekind C, Compton CC, Greene FL and Sobin LH: TNM residual tumor classification revisited. Cancer 94: 2511-2516, 2002.

2. Brenner H, Kloor M and Pox CP: Colorectal cancer. Lancet 383 : $1490-1502,2014$

3. André T, Boni C, Mounedji-Boudiaf L, Navarro $M$, Tabernero J, Hickish T, Topham C, Zaninelli M, Clingan P, Bridgewater $\mathrm{J}$, et al; Multicenter International Study of Oxaliplatin/5-Fluorouracil/Leucovorin in the Adjuvant Treatment of Colon Cancer (MOSAIC) Investigators: Oxaliplatin, fluorouracil, and leucovorin as adjuvant treatment for colon cancer. N Engl J Med 350: 2343-2351, 2004.

4. Sobrero A, Lonardi S, Rosati G, Di Bartolomeo M, Ronzoni M, Pella N, Scartozzi M, Banzi M, Zampino MG, Pasini F, et al: FOLFOX or CAPOX in Stage II to III Colon Cancer: Efficacy Results of the Italian Three or Six Colon Adjuvant Trial. J Clin Oncol 36: 1478-1485, 2018

5. Gill S, Meyerhardt JA, Arun M and Veenstra CM: Translating IDEA to Practice and Beyond: Managing Stage II and III Colon Cancer. Am Soc Clin Oncol Educ Book 39: 226-235, 2019.

6. Galon J, Costes A, Sanchez-Cabo F, Kirilovsky A, Mlecnik B, Lagorce-Pagès $\mathrm{C}$, Tosolini $\mathrm{M}$, Camus $\mathrm{M}$, Berger $\mathrm{A}$, Wind $\mathrm{P}$, et al: Type, density, and location of immune cells within human colorectal tumors predict clinical outcome. Science 313 1960-1964, 2006

7. Broussard EK and Disis ML: TNM staging in colorectal cancer: $\mathrm{T}$ is for $\mathrm{T}$ cell and $\mathrm{M}$ is for memory. $\mathrm{J}$ Clin Oncol 29: 601-603, 2011.

8. Fridman WH, Pagès F, Sautès-Fridman C and Galon J: The immune contexture in human tumours: Impact on clinical outcome. Nat Rev Cancer 12: 298-306, 2012.

9. Galon J, Angell HK, Bedognetti D and Marincola FM: The continuum of cancer immunosurveillance: Prognostic, predictive, and mechanistic signatures. Immunity 39: 11-26, 2013.

10. Mei Z, Liu Y, Liu C, Cui A, Liang Z, Wang G, Peng H, Cui L and Li C: Tumour-infiltrating inflammation and prognosis in colorectal cancer: Systematic review and meta-analysis. Br J Cancer 110: 1595-1605, 2014.

11. Mlecnik B, Tosolini M, Kirilovsky A, Berger A, Bindea G, Meatchi T, Bruneval P, Trajanoski Z, Fridman WH, Pagès F, et al: Histopathologic-based prognostic factors of colorectal cancers are associated with the state of the local immune reaction. J Clin Oncol 29: 610-618, 2011.

12. Van den Eynde M, Mlecnik B, Bindea G, Fredriksen T, Church SE, Lafontaine L, Haicheur N, Marliot F, Angelova M, Vasaturo A, et al: The Link between the Multiverse of Immune Microenvironments in Metastases and the Survival of Colorectal Cancer Patients. Cancer Cell 34: 1012-1026.e3, 2018.

13. Pagès F, Mlecnik B, Marliot F, Bindea G, Ou F-S, Bifulco C Lugli A, Zlobec I, Rau TT, Berger MD, et al: International validation of the consensus Immunoscore for the classification of colon cancer: A prognostic and accuracy study. Lancet 391: 2128-2139, 2018.

14. Kerkar SP and Restifo NP: Cellular constituents of immune escape within the tumor microenvironment. Cancer Res 72 : $3125-3130,2012$

15. Chen L: Co-inhibitory molecules of the B7-CD28 family in the control of T-cell immunity. Nat Rev Immunol 4: 336-347, 2004.

16. Mahoney KM, Rennert PD and Freeman GJ: Combination cancer immunotherapy and new immunomodulatory targets. Nat Rev Drug Discov 14: 561-584, 2015.

17. Bethmann D, Feng Z and Fox BA: Immunoprofiling as a predictor of patient's response to cancer therapy-promises and challenges. Curr Opin Immunol 45: 60-72, 2017

18. Salama P, Phillips M, Grieu F, Morris M, Zeps N, Joseph D, Platell $\mathrm{C}$ and Iacopetta B: Tumor-infiltrating FOXP3 $3^{+} \mathrm{T}$ regulatory cells show strong prognostic significance in colorectal cancer. J Clin Oncol 27: 186-192, 2009.

19. Sinicrope FA, Rego RL, Ansell SM, Knutson KL, Foster NR and Sargent DJ: Intraepithelial effector $\left(\mathrm{CD}^{+}\right) /$regulatory $\left(\mathrm{FoxP}^{+}\right)$T-cell ratio predicts a clinical outcome of human colon carcinoma. Gastroenterology 137: 1270-1279, 2009.
20. deLeeuw RJ, Kost SE, Kakal JA and Nelson BH: The prognostic value of FoxP $3^{+}$tumor-infiltrating lymphocytes in cancer: A critical review of the literature. Clin Cancer Res 18: 3022-3029, 2012.

21. Saito T, Nishikawa H, Wada H, Nagano Y, Sugiyama D, Atarashi K, Maeda Y, Hamaguchi M, Ohkura N, Sato E, et al: Two FOXP3(+)CD4(+) T cell subpopulations distinctly control the prognosis of colorectal cancers. Nat Med 22: 679-684, 2016.

22. Gabrilovich DI: Myeloid-Derived Suppressor Cells. Cancer Immunol Res 5: 3-8, 2017.

23. Shipp C, Speigl L, Janssen N, Martens A and Pawelec G: A clinical and biological perspective of human myeloid-derived suppressor cells in cancer. Cell Mol Life Sci 73: 4043-4061, 2016

24. Duan J, Xie Y, Qu L, Wang L, Zhou S, Wang Y, Fan Z, Yang S and Jiao S: A nomogram-based immunoprofile predicts overall survival for previously untreated patients with esophageal squamous cell carcinoma after esophagectomy. J Immunother Cancer 6: 100, 2018

25. Li F, Zhao Y, Wei L, Li S and Liu J: Tumor-infiltrating Treg, MDSC, and IDO expression associated with outcomes of neoadjuvant chemotherapy of breast cancer. Cancer Biol Ther 19: 695-705, 2018.

26. Chen L and Han X: Anti-PD-1/PD-L1 therapy of human cancer: Past, present, and future. J Clin Invest 125: 3384-3391, 2015.

27. Gong J, Chehrazi-Raffle A, Reddi S and Salgia R: Development of PD-1 and PD-L1 inhibitors as a form of cancer immunotherapy: A comprehensive review of registration trials and future considerations. J Immunother Cancer 6: 8, 2018.

28. Ganesh K, Stadler ZK, Cercek A, Mendelsohn RB, Shia J, Segal NH and Diaz LA Jr: Immunotherapy in colorectal cancer: Rationale, challenges and potential. Nat Rev Gastroenterol Hepatol 16: 361-375, 2019.

29. Marin-Acevedo JA, Dholaria B, Soyano AE, Knutson KL, Chumsri S and Lou Y: Next generation of immune checkpoint therapy in cancer: New developments and challenges. J Hematol Oncol 11: 39, 2018.

30. Zhai L, Ladomersky E, Lenzen A, Nguyen B, Patel R, Lauing KL, $\mathrm{Wu} \mathrm{M}$ and Wainwright DA: IDO1 in cancer: A Gemini of immune checkpoints. Cell Mol Immunol 15: 447-457, 2018.

31. Wang Y, Li J, Xia Y, Gong R, Wang K, Yan Z, Wan X, Liu G, Wu D, Shi L, et al: Prognostic nomogram for intrahepatic cholangiocarcinoma after partial hepatectomy. J Clin Oncol 31: $1188-1195,2013$

32. Fong Y: Textbook Outcome Nomograms as Multivariate Clinical Tools for Building Cancer Treatment Pathways and Prognosticating Outcomes. JAMA Surg 154: e190572, 2019.

33. Camp RL, Dolled-Filhart M and Rimm DL; RL C: X-tile: A new bio-informatics tool for biomarker assessment and outcome-based cut-point optimization. Clin Cancer Res 10: 7252-7259, 2004

34. Stack EC, Wang C, Roman KA and Hoyt CC: Multiplexed immunohistochemistry, imaging, and quantitation: A review, with an assessment of Tyramide signal amplification, multispectral imaging and multiplex analysis. Methods 70: 46-58, 2014

35. Zhang B, Wang Z, Wu L, Zhang M, Li W, Ding J, Zhu J, Wei H and Zhao K: Circulating and tumor-infiltrating myeloid-derived suppressor cells in patients with colorectal carcinoma. PLoS One 8: e57114, 2013.

36. Hanahan D and Weinberg RA: Hallmarks of cancer: The next generation. Cell 144: 646-674, 2011.

37. Chen DS and Mellman I: Elements of cancer immunity and the cancer-immune set point. Nature 541: 321-330, 2017.

38. Golstein P and Griffiths GM: An early history of T cell-mediated cytotoxicity. Nat Rev Immunol 18: 527-535, 2018

39. Pagès F, Kirilovsky A, Mlecnik B, Asslaber M, Tosolini M, Bindea G, Lagorce C, Wind P, Marliot F, Bruneval P, et al: In situ cytotoxic and memory $\mathrm{T}$ cells predict outcome in patients with early-stage colorectal cancer. J Clin Oncol 27: 5944-5951, 2009.

40. Feng Z, Bethmann D, Kappler M, Ballesteros-Merino C, Eckert A, Bell RB, Cheng A, Bui T, Leidner R, Urba WJ, et al: Multiparametric immune profiling in $\mathrm{HPV}^{-}$oral squamous cell cancer. JCI Insight 2: 2, 2017.

41. Stromnes IM, Hulbert A, Pierce RH, Greenberg PD and Hingorani SR: T-cell Localization, Activation, and Clonal Expansion in Human Pancreatic Ductal Adenocarcinoma. Cancer Immunol Res 5: 978-991, 2017.

42. Han Y, Liu D and Li L: PD-1/PD-L1 pathway: Current researches in cancer. Am J Cancer Res 10: 727-742, 2020.

43. Solinas C, De Silva P, Bron D, Willard-Gallo K and Sangiolo D: Significance of TIM3 expression in cancer: From biology to the clinic. Semin Oncol 46: 372-379, 2019. 
44. Webb GJ, Hirschfield GM and Lane PJ: OX40, OX40L and Autoimmunity: A Comprehensive Review. Clin Rev Allergy Immunol 50: 312-332, 2016.

45. Weixler B, Cremonesi E, Sorge R, Muraro MG, Delko T, Nebiker CA, Däster S, Governa V, Amicarella F, Soysal SD, et al: OX40 expression enhances the prognostic significance of CD8 positive lymphocyte infiltration in colorectal cancer. Oncotarget 6: 37588-37599, 2015.

46. Xiao Z, Mayer AT, Nobashi TW and Gambhir SS: ICOS Is an Indicator of T-cell-Mediated Response to Cancer Immunotherapy. Cancer Res 80: 3023-3032, 2020.

47. Nakano M, Ito M, Tanaka R, Yamaguchi K, Ariyama H, Mitsugi K, Yoshihiro T, Ohmura H, Tsuruta N, Hanamura F, et al: PD $-1^{+}$TIM $-3^{+}$T cells in malignant ascites predict prognosis of gastrointestinal cancer. Cancer Sci 109: 2986-2992, 2018.

48. Tay RE, Richardson EK and Toh HC: Revisiting the role of $\mathrm{CD} 4^{+} \mathrm{T}$ cells in cancer immunotherapy-new insights into old paradigms. Cancer Gene Ther 28: 5-17, 2021.

49. Merritt CR, Ong GT, Church SE, Barker K, Danaher P, Geiss G, Hoang M, Jung J, Liang Y, McKay-Fleisch J, et al: Multiplex digital spatial profiling of proteins and RNA in fixed tissue. Nat Biotechnol 38: 586-599, 2020.
50. Kumar V, Patel S, Tcyganov E and Gabrilovich DI: The Nature of Myeloid-Derived Suppressor Cells in the Tumor Microenvironment. Trends Immunol 37: 208-220, 2016.

51. Sun HL, Zhou X, Xue YF, Wang K, Shen YF, Mao JJ, Guo HF and Miao ZN: Increased frequency and clinical significance of myeloid-derived suppressor cells in human colorectal carcinoma. World J Gastroenterol 18: 3303-3309, 2012.

52. Limagne E, Euvrard R, Thibaudin M, Rébé C, Derangère V, Chevriaux A, Boidot R, Végran F, Bonnefoy N, Vincent J, et al: Accumulation of MDSC and Th17 Cells in Patients with Metastatic Colorectal Cancer Predicts the Efficacy of a FOLFOX-Bevacizumab Drug Treatment Regimen. Cancer Res 76: 5241-5252, 2016

53. Nagaraj S, Nelson A, Youn JI, Cheng P, Quiceno D and Gabrilovich DI: Antigen-specific CD4(+) T cells regulate function of myeloid-derived suppressor cells in cancer via retrograde MHC class II signaling. Cancer Res 72: 928-938, 2012.

(i) $\ominus$ This work is licensed under a Creative Commons c) Attribution-NonCommercial-NoDerivatives 4.0 International (CC BY-NC-ND 4.0) License. 